\title{
Purchasing power parity in ASEAN+3: an application of panel unit root tests
}

\author{
Darja Boršič \\ University of Maribor, Faculty of Economics and Business, Slovenia \\ darja.borsic@um.si \\ Jani Bekő \\ University of Maribor, Faculty of Economics and Business, Slovenia \\ jani.beko@um.si
}

\begin{abstract}
The paper assesses the existence of purchasing power parity (PPP) in ASEAN+3 economies taking into account EUR and USD as reference currencies. The research refers to the period from January 2000 to June 2017 and there are three points of view: we tested the period as a whole and then the pre-crisis period and the postcrisis period regarding the structural break due to the economic crisis. The evaluated economies include Brunei, Cambodia, China, Indonesia, Japan, Korea, Laos, Malaysia, Myanmar, the Philippines, Singapore, Thailand and Vietnam. A range of panel unit root tests are applied, covering the Levin, Lin and Chu test, the Breitung test, the Im, Pesaran and Shin test, the ADF-Fisher test and the PP-Fisher test. In cases where the unit root is rejected, the validity of PPP is confirmed. However, our results are ambiguous and depend on the selection of the base currency, the time period observed and on the choice of the methodology.
\end{abstract}

Keywords: ASEAN+3, EUR, panel unit root tests, purchasing power parity, real exchange rates, USD.

JEL classification: C33, F31, P22.

DOI:10.2478/crebss-2018-0004

Received: April 20, 2018

Accepted: June 14, 2018

\section{Acknowledgement}

The authors acknowledge the financial support from the Slovenian Research Agency (research core funding No. P5-0027).

\section{Introduction}

An irreplaceable element in monetary macroeconomic models for open economies is the concept of purchasing power parity (PPP). The PPP theory implies that exchange rate changes are driven based on the arbitrage, by shifts in relative prices, thus making potential deviations of real exchange rates from the PPPequilibrium levels only as a short-run phenomenon. Consequently, the PPP proposition is one of the most frequently examined topics in empirical macroeconomics. In recent years, we have witnessed an increase of papers testing the validity of this exchange rate theory also for Asian economies. The growing 
power of Asian countries in global production and investments, their expansion in international trade and tight economic cooperation are the main factors that motivate researchers to apply PPP on these economies. However, despite of employing different estimation techniques, data samples, including various groups of Asian countries, studies provided mixed empirical results, which strengthen the PPP puzzle.

In this research, we aim to contribute to the empirics of PPP in three ways. First, the PPP model is tested on the panel that covers selected 10 countries of the Association of Southeast Asian Nations (ASEAN), with China, Japan and South Korea. Second, an array of panel unit root tests is used on new data series in order to examine among others the validity of PPP after the outbreak of Great Recession. Third, all the unit root tests are performed simultaneously for USD and EUR rates. The elementary research hypothesis in the presents study is that the PPP concept holds for the selected group of Asian countries. Our paper is organized as follows. The next chapter contains a short literature review, while the basic theory of PPP, data properties and the econometric methodology are outlined in the following chapter. After that, the empirical results are presented. The key findings of our analysis are highlighted in the concluding part of the paper.

\section{Literature Review}

Arize, Malindretos and Ghosh (2015) tested the cointegration characteristics of exchange rates and prices of 27 Asian countries against the USD and found support for PPP in significant number of economies. Recently, Choji and Sek (2017) examined five ASEAN members with threshold cointegration tests but detected long run PPP in only two cases. The stationarity of real exchange rates on the USD is proven in seven out of eight Asian economies in the study prepared by Zhou and Kutan (2011).

On the other hand, Chang, Zhang and Liu (2010) were able to reject the unit root hypothesis for no more than four countries' real exchange rates among eight ASEAN members only after the panel SURKSS tests are applied with respect to the USD and the JPY. Based on nonlinear unit root tests using the USD as the numeraire, Chang, Lee and Liu (2012) also report that PPP is relevant merely for three among eight of the ASEAN economies. In addition, the PPP proposition is evaluated for the group of ASEAN-5 with a wide range of advanced panel unit root tests and cointegration analysis by Munir and Kok (2015). The evidence on PPP, based on stationarity properties of real exchange rates with respect to the USD, is in this paper fragmentary, substantially more support for the theory is gained from the employment of panel cointegration test. Munir and Kok (2015) advocate to extend the PPP testing by using different numeraire currencies. With the threshold cointegration technique incorporating asymmetric adjustments Lu and Chang (2011) provided evidence on long-run PPP for China. Paper by Lau et al. (2012) also confirms the PPP for four ASEAN countries when China acts as a base country, which at the same time indicates the significance of trade and financial links between economies in the Asian region.

\section{The PPP theory and research methodology}

The empirical characteristics of purchasing power parity (PPP) can be denoted as (Froot, Rogoff, 1995):

$$
e_{\dagger}=\alpha_{0}+\alpha_{1} p_{\dagger}+\alpha_{2} p_{\dagger}^{*}+\xi_{\dagger}
$$

where et presents nominal exchange rates expressed as the price of the foreign currency in the domestic currency units; $p_{t}$ is abbreviation for domestic price index 
and $\mathrm{p}_{t}{ }^{*}$ for foreign price index. The error term $\xi_{\dagger}$ denotes deviations from PPP. The considered variables are given as logarithms. According to the strict version of PPP the symmetry restriction requires that $\alpha_{0}=0$, while $\alpha_{1}$ and $\alpha_{2}$ are equal in absolute terms, and proportionality restriction demands that $\alpha_{0}=0, \alpha_{1}=1$ and $\alpha_{2}=-1$.

The empirical analysis is based on monthly data ranging from January 2000 to June 2017 for the ASEAN+3 economies, including Brunei, Cambodia, China, Indonesia, Japan, Korea, Laos, Malaysia, Myanmar, Philippines, Singapore, Thailand and Vietnam. The consumer price indices $(2010=100)$ and the monthly averages of nominal exchange rates with reference currencies of EUR and USD were gathered from IMF International Financial Statistics. The empirical analysis was undertaken in three parts, taking into account the whole observed period (1), the pre-economic crisis period (2) ranging from January 2000 to December 2007, and the posteconomic crisis period (3) ranging from July 2009 to June 2017. The sub-periods where determined following the NBER (2012) methodology for defining the US business cycles.

In the empirical analysis, we assess the features of real exchange rates, which are in line with the strict version of PPP in Equation 1. The changes in relative prices are supposed to be offset by the movements in nominal exchange rates. Consequently, the real exchange rates should remain constant over the long period. Also, their time series should not exhibit unit roots, they are expected to be stationary (Parikh, Wakerley, 2000). This paper applies panel unit root tests in order to find evidence in favour of PPP.

The model considers the AR(1) process for panel data may be described as follows:

$$
y_{i, t}=\rho_{i} y_{i, t-1}+X_{i, t} \delta_{i}+\varepsilon_{i, t}
$$

where the index $i$ denotes $N$ cross-section units for the periods for $t=1,2, \ldots, T_{i}, X_{i, t}$ are exogenous variables in the model, pi are autoregressive coefficients. Here the errors $(\varepsilon, t)$ are jointly independent idiosyncratic disturbances. When the absolute value of $\rho_{i}$ is less than $1, y_{i}$ is weakly stationary. When the absolute value of $\rho_{i}$ is $1, y_{i}$ contains a unit root. The panel unit root tests in our analysis differ in two assumptions about the $\rho_{i}$ in panel unit root tests. Levin, Lin and Chu (2002) and Breitung (2000) approaches consider common unit root processes, with common autoregressive coefficients across cross-sections ( $\left.\rho_{i}=\rho\right)$ for all i. On the other hand, the authors Im, Pesaran and Shin (2003), considered the Fisher modes of the augmented Dickey-Fuller (ADF) and the Phillips-Perron (PP) test, Maddala, Wu (1999) and Choi (2001), testing procedures dealing with the processes of the individual unit root, where pi vary across crosssections.

\section{Description of the Levin, Lin and Chu test, and the Breitung test}

The Levin, Lin and Chu (2002) test takes into account the following ADF specification:

$$
\Delta y_{i, t}=\alpha y_{i, t-1}+\sum_{j=1}^{p_{i}} \beta_{i, j} \Delta y_{i, t-j}+X_{i, t}^{\prime} \delta+\varepsilon_{i, t},
$$

where a common $a=\rho-1$ is assumed. The order of the lag for difference terms $\left(p_{i}\right)$ is changing over the cross-sections. The null hypothesis $\left(\mathrm{H}_{0}: \mathrm{a}=0\right)$ implies that there is $a$ unit root. While the alternative hypothesis $\left(H_{1}: a<0\right)$ implies stationarity. In the Levin, Lin and Chu (2002) approach the first step requires an assessment of auxiliary regressions of $\Delta y_{i, t}$ and $y_{i, t}$ on lagged terms $\Delta y_{i, t-j}$ and on exogenous variables $x_{i t}$. The residuals, which are denoted by $(\sim)$ are used as proxies for $\Delta y_{i, t}$ and $y_{i, t .}$ Further, the term $a$ is calculated using the pooled equation:

$$
\Delta \tilde{y}_{i, t}=\alpha \tilde{y}_{i, t-1}+\eta_{i, t} .
$$


Due to the fact that the common t-statistic, which was used for testing the hypothesis with $\hat{\alpha}=0$, is diverging to negative infinity, the authors Levin, Lin and Chu (2002) developed the modified t statistics (t* in Equation 5). This modified t statictic is shown to be asymptotically normally distributed. Now, the following statistic:

$$
t^{*}=\frac{1}{\sigma^{*}}\left(t-N T \cdot \hat{S}_{N} \hat{\sigma}^{-2} \hat{\sigma}_{\alpha} \mu^{*}\right)
$$

where $\mu^{*}$ and $\sigma^{*}$ are the terms used for alterations for the mean and the standard deviation calculated by Levin, Lin and Chu (2002), $\hat{\sigma}_{\alpha}$ is the standard error of $\hat{\alpha}, \hat{\sigma}^{2}$ is the estimated variance of the residuals from equation (4) and $\hat{S}_{N}$ denotes the mean of individual ratios of standard deviations for all the runs, long and short. $\hat{S}_{N}$ is estimated by the kernel-based techniques. According to Levin, Lin and Chu (2002), we applied Bartlett kernel, Parzen kernel and quadratic spectral kernel. For checking the results robustness, we also considered regression (3) amplified with the distinct deterministic linear trend models. The Schwarz information criterion was used in selecting the number of lags in each of the cross-section ADF regressions ( $\left.\mathrm{p}_{\mathrm{i}}\right)$.

The Breitung test is similar to Lin, Levin and Chu test. It estimates auxiliary regressions of $\Delta y_{i, t}$ and $y_{i, t}$ on lagged terms $\Delta y_{i, t-j}$ only, while proxies are transformed and detrended $\left(\Delta y_{i t}{ }^{*}\right)$. Panel proxy equation is used to estimate the persistence parameter $a$ :

$$
\Delta y_{i t}{ }^{*}=a y_{i t-1}{ }^{*}+v_{\text {it }} \text {. }
$$

According to Breitung (2000) under the null hypothesis, the estimate of the persistence parameter $a$ is asymptotically normally distributed.

\section{Description of the Im, Pesaran and Shin test}

Im, Pesaran and Shin (2003) take into account the processes of the individual unit root and estimate the individual ADF regression for each of the cross-sections:

$$
\Delta y_{i, t}=\alpha y_{i, t-1}+\sum_{j=1}^{p_{i}} \beta_{i, j} \Delta y_{i, t-j}+X_{i, t}^{\prime} \delta+\varepsilon_{i, t}
$$

where the null hypothesis is fixed as follows:

$$
H_{0}: \alpha_{i}=0, \text { for all } \mathrm{i} \text {. }
$$

The working hypothesis is set as:

$$
H_{1}:\left\{\begin{array}{lll}
\alpha_{i}=0 & \text { for } \mathrm{i}=1,2, \ldots, \mathrm{N}_{1} \\
\alpha_{i}<0 & \text { for } \mathrm{i}=\mathrm{N}_{1}+1, \ldots, \mathrm{N}
\end{array} .\right.
$$

$\bar{t}$ stands for the average of the t-statistics for $a_{i}$ from individual ADF regressions:

$$
\bar{t}=\frac{1}{N} \sum_{i=1}^{N} t_{i} .
$$

The authors Im, Pesaran and Shin (2003) decided to standardize the $\bar{t}$-statistic using the new symbol $W$. The new statistic $W$ is asymptotically approaching the normality.

\section{Description of the Fisher ADF and the Fisher PP tests}

Taking into account the results of Fischer (1932), Maddala and WU (1999) and Choi (2001) developed the tests that integrate the individual $p$-values. $\pi i$ denotes the $p$ value from the individual unit root test ofr the cross-section i. According to Hurlin (2010) discovered that the respective $p$-values are following the uniform [0, 1] distribution. Maddala and Wu (1999) define their own statistic given as:

$$
\chi^{2}=-2 \sum_{i=1}^{N} \log \left(\pi_{i}\right)
$$


and prove that it has an asymptotic $\mathrm{X}^{2}$-distribution with $2 \mathrm{~N}$ degrees of freedom. Choi (2001) defines a similar Z statistic:

$$
Z=-\frac{\sum_{i=1}^{N} \log \left(\pi_{i}\right)+N}{\sqrt{N}}
$$

The null and the alternative hypotheses are the same as for the Im, Pesaran and Shin test described I Equations 8 and 9. Under the null hypothesis, the Z-statistic is following the normal distribution.

\section{Results and Discussion}

As shown in Table 1, in the whole observed period for the EUR exchange rates, the null hypothesis for the unit root could be rejected. The PPP theory may be confirmed in the panel of the observed countries only if the individual effects and the linear deterministic trends are taken into account. In the pre-crisis period, the null hypothesis should be rejected at $10 \%$ level of significance only for the case of the individual effects, too. While for the post-crisis period with the EUR as the reference currency, the hypothesis for PPP is confirmed when only individual effects are taken into account. Regarding the USD as the reference currency, the results confirm the null hypothesis and thus reject the PPP validity for the whole period as well as for the pre-crisis period. While in the post-crisis period, the null is rejected and PPP confirmed for all kernel-based techniques and regardless of the deterministic component applied.

Table 1 Results of Levin, Lin and Chu test

\begin{tabular}{|c|c|c|c|c|c|c|c|}
\hline \multirow{3}{*}{$\begin{array}{l}\frac{0}{0} \\
\frac{\hat{Q}}{0} \\
\stackrel{\infty}{0}\end{array}$} & \multirow{3}{*}{ 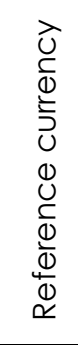 } & \multicolumn{2}{|c|}{ Bartlett kernel } & \multicolumn{2}{|c|}{ Parzen kernel } & \multicolumn{2}{|c|}{ Quadratic spectral kernel } \\
\hline & & $\begin{array}{l}\text { Individual } \\
\text { effects }\end{array}$ & $\begin{array}{l}\text { Individual } \\
\text { effects and } \\
\text { individual } \\
\text { linear trends }\end{array}$ & $\begin{array}{l}\text { Individual } \\
\text { effects }\end{array}$ & $\begin{array}{l}\text { Individual } \\
\text { effects and } \\
\text { individual } \\
\text { linear trends }\end{array}$ & $\begin{array}{l}\text { Individual } \\
\text { effects }\end{array}$ & $\begin{array}{l}\text { Individual } \\
\text { effects and } \\
\text { individual } \\
\text { linear trends }\end{array}$ \\
\hline & & $\begin{array}{c}t^{*} \\
\text { (p-value) } \\
\text { [no. of } \\
\text { lags] }\end{array}$ & $\begin{array}{c}t^{*} \\
\text { (p-value) } \\
\text { [no. of lags] }\end{array}$ & $\begin{array}{c}t^{*} \\
\text { (p-value) } \\
\text { [no. of lags] }\end{array}$ & $\begin{array}{c}t^{*} \\
\text { (p-value) } \\
\text { [no. of lags] }\end{array}$ & $\begin{array}{c}t^{*} \\
\text { (p-value) } \\
\text { [no. of } \\
\text { lags] }\end{array}$ & $\begin{array}{c}t^{*} \\
\text { (p-value) } \\
\text { [no. of lags] }\end{array}$ \\
\hline \multirow{2}{*}{ 方交 } & EUR & $\begin{array}{c}0.11252 \\
(0.5448) \\
{[0-2]}\end{array}$ & $\begin{array}{c}-2.11559 \\
(0.0172) \\
{[0-2]}\end{array}$ & $\begin{array}{c}0.24110 \\
(0.5953) \\
{[0-2]}\end{array}$ & $\begin{array}{c}-1.90672 \\
(0.0283) \\
{[0-2]}\end{array}$ & $\begin{array}{c}0.21001 \\
(0.5832) \\
{[0-2]}\end{array}$ & $\begin{array}{c}-1.87136 \\
(0.0306) \\
{[0-2]}\end{array}$ \\
\hline & USD & $\begin{array}{c}0.54694 \\
(0.7078) \\
{[0-2]}\end{array}$ & $\begin{array}{c}-0.41399 \\
(0.3394) \\
{[0-2]}\end{array}$ & $\begin{array}{c}0.64482 \\
(0.7405) \\
{[0-2]}\end{array}$ & $\begin{array}{c}-0.08532 \\
(0.4660) \\
{[0-2]}\end{array}$ & $\begin{array}{c}0.59772 \\
(0.7250) \\
{[0-2]} \\
\end{array}$ & $\begin{array}{c}-0.22125 \\
(0.4124) \\
{[0-2]} \\
\end{array}$ \\
\hline \multirow{2}{*}{ 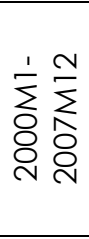 } & EUR & $\begin{array}{c}-1.29066 \\
(0.0984) \\
{[0-1]} \\
\end{array}$ & $\begin{array}{c}0.24298 \\
(0.5960) \\
{[0-1]}\end{array}$ & $\begin{array}{c}-1.35421 \\
(0.0878) \\
{[0-1]} \\
\end{array}$ & $\begin{array}{c}0.04030 \\
(0.5161) \\
{[0-1]} \\
\end{array}$ & $\begin{array}{c}-1.36478 \\
(0.0862) \\
{[0-1]}\end{array}$ & $\begin{array}{c}0.02653 \\
(0.5106) \\
{[0-1]} \\
\end{array}$ \\
\hline & USD & $\begin{array}{c}2.09301 \\
(0.9818) \\
{[0-4]}\end{array}$ & $\begin{array}{c}-0.43032 \\
(0.3335) \\
{[0-4]}\end{array}$ & $\begin{array}{c}2.15218 \\
(0.9843) \\
{[0-4]}\end{array}$ & $\begin{array}{c}-0.37037 \\
(0.3556) \\
{[0-4]}\end{array}$ & $\begin{array}{c}2.09781 \\
(0.9820) \\
{[0-4]}\end{array}$ & $\begin{array}{c}-0.21909 \\
(0.4133) \\
{[0-4]}\end{array}$ \\
\hline \multirow{2}{*}{ 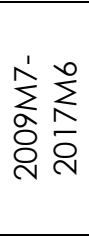 } & EUR & $\begin{array}{c}-3.21396 \\
(0.0007) \\
{[0-1]}\end{array}$ & $\begin{array}{c}0.45711 \\
(0.6762) \\
{[0-1]}\end{array}$ & $\begin{array}{c}-3.16081 \\
(0.0008) \\
{[0-1]}\end{array}$ & $\begin{array}{c}0.57244 \\
(0.7165) \\
{[0-1]}\end{array}$ & $\begin{array}{c}-3.16640 \\
(0.0008) \\
{[0-1]}\end{array}$ & $\begin{array}{c}0.56188 \\
(0.7129) \\
{[0-1]}\end{array}$ \\
\hline & USD & $\begin{array}{c}-3.19857 \\
(0.0007) \\
{[0-3]}\end{array}$ & $\begin{array}{c}-1.86175 \\
(0.0313) \\
{[0-2]}\end{array}$ & $\begin{array}{c}-3.08431 \\
(0.0010) \\
{[0-3]}\end{array}$ & $\begin{array}{c}-1.85279 \\
(0.0320) \\
{[0-2]}\end{array}$ & $\begin{array}{c}-3.11641 \\
(0.0009) \\
{[0-3]}\end{array}$ & $\begin{array}{c}-1.75211 \\
(0.0399) \\
{[0-2]}\end{array}$ \\
\hline
\end{tabular}

Table 2 presents the results of the Breitung test, where null of a unit root could not be rejected at $5 \%$ significance level no matter which reference currency neither the 
information criteria was taken into account. Thus, the validity of PPP cannot be confirmed. The closest to PPP is the case of EUR as the reference currency in the precrisis period with Hannan-Quinn information criteria for the lag selection, where the null can be rejected at $10 \%$ significance level.

Table 2 Results of Breitung test

\begin{tabular}{|c|c|c|c|c|}
\hline \multirow{3}{*}{$\frac{\frac{0}{O}}{\frac{\varrho}{D}}$} & \multirow{3}{*}{ 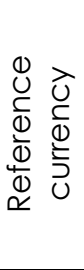 } & $\begin{array}{c}\text { Schwarz information } \\
\text { criterion }\end{array}$ & Akaike information criterion & $\begin{array}{l}\text { Hannan-Qiunn information } \\
\text { criterion }\end{array}$ \\
\hline & & $\begin{array}{l}\text { Individual effects and } \\
\text { individual linear trends }\end{array}$ & $\begin{array}{l}\text { Individual effects and } \\
\text { individual linear trends }\end{array}$ & $\begin{array}{l}\text { Individual effects and } \\
\text { individual linear trends }\end{array}$ \\
\hline & & $\begin{array}{c}\text { t-stat } \\
\text { (p-value) } \\
\text { [no. of lags] }\end{array}$ & $\begin{array}{c}\text { t-stat } \\
\text { (p-value) } \\
\text { [no. of lags] }\end{array}$ & $\begin{array}{c}\text { t-stat } \\
\text { (p-value) } \\
\text { [no. of lags] }\end{array}$ \\
\hline \multirow{2}{*}{ 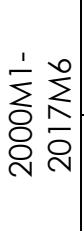 } & EUR & $\begin{array}{c}-0.15457 \\
(0.4386) \\
{[0-2]}\end{array}$ & $\begin{array}{c}-0.09767 \\
(0.4611) \\
{[0-13]}\end{array}$ & $\begin{array}{c}-0.14660 \\
(0.4417) \\
{[0-2]}\end{array}$ \\
\hline & USD & $\begin{array}{c}0.39668 \\
(0.6542) \\
{[0-2]}\end{array}$ & $\begin{array}{c}-0.06786 \\
10.4729 \\
{[0-12]}\end{array}$ & $\begin{array}{c}0.01640 \\
(0.5065) \\
{[0-12]}\end{array}$ \\
\hline \multirow{2}{*}{ 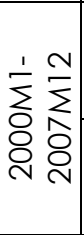 } & EUR & $\begin{array}{c}-1.16673 \\
(0.1217) \\
{[0-1]}\end{array}$ & $\begin{array}{c}-1.09443 \\
(0.1369) \\
{[0-11]}\end{array}$ & $\begin{array}{c}-1.32669 \\
(0.0923) \\
{[0-9]}\end{array}$ \\
\hline & USD & $\begin{array}{c}5.01460 \\
(1.0000) \\
{[0-4]}\end{array}$ & $\begin{array}{c}5.63700 \\
(1.0000) \\
{[0-10]}\end{array}$ & $\begin{array}{c}5.88935 \\
(1.0000) \\
{[0-10]}\end{array}$ \\
\hline \multirow{2}{*}{ 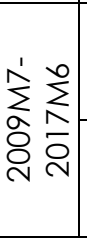 } & EUR & $\begin{array}{c}0.31629 \\
(0.6241) \\
{[0-1]}\end{array}$ & $\begin{array}{c}-0.35685 \\
(0.3606) \\
{[0-10]}\end{array}$ & $\begin{array}{c}-0.11387 \\
(0.4547) \\
{[0-1]}\end{array}$ \\
\hline & USD & $\begin{array}{c}3.68108 \\
(0.9999) \\
{[0-2]}\end{array}$ & $\begin{array}{c}4.13232 \\
(1.0000) \\
{[0-9]}\end{array}$ & $\begin{array}{c}3.06602 \\
(0.9989) \\
{[0-3]}\end{array}$ \\
\hline
\end{tabular}

Table 3 Results of Im, Pesaran and Shin test

\begin{tabular}{|c|c|c|c|c|c|c|c|}
\hline \multirow{3}{*}{$\begin{array}{l}\frac{0}{0} \\
\frac{0}{0} \\
\stackrel{\sim}{\circ}\end{array}$} & \multirow{3}{*}{ 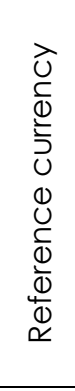 } & \multicolumn{2}{|c|}{$\begin{array}{c}\text { Schwarz information } \\
\text { criterion }\end{array}$} & \multicolumn{2}{|c|}{ Akaike information criterion } & \multicolumn{2}{|c|}{$\begin{array}{l}\text { Hannan-Qiunn information } \\
\text { criterion }\end{array}$} \\
\hline & & $\begin{array}{l}\text { Individual } \\
\text { effects }\end{array}$ & $\begin{array}{l}\text { Individual } \\
\text { effects and } \\
\text { individual } \\
\text { linear trends }\end{array}$ & $\begin{array}{l}\text { Individual } \\
\text { effects }\end{array}$ & $\begin{array}{l}\text { Individual } \\
\text { effects and } \\
\text { individual } \\
\text { linear trends }\end{array}$ & $\begin{array}{l}\text { Individual } \\
\text { effects }\end{array}$ & $\begin{array}{l}\text { Individual } \\
\text { effects and } \\
\text { individual } \\
\text { linear trends }\end{array}$ \\
\hline & & $\begin{array}{c}\text { W-stat } \\
\text { (p-value) } \\
\text { [no. of } \\
\text { lags] }\end{array}$ & $\begin{array}{c}\text { W-stat } \\
\text { (p-value) } \\
\text { [no. of lags] }\end{array}$ & $\begin{array}{c}\text { W-stat } \\
\text { (p-value) } \\
\text { [no. of } \\
\text { lags] }\end{array}$ & $\begin{array}{c}\text { W-stat } \\
\text { (p-value) } \\
\text { [no. of lags] }\end{array}$ & $\begin{array}{c}\text { W-stat } \\
\text { (p-value) } \\
\text { [no. of } \\
\text { lags] }\end{array}$ & $\begin{array}{c}\text { W-stat } \\
\text { (p-value) } \\
\text { [no. of lags] }\end{array}$ \\
\hline \multirow{2}{*}{ 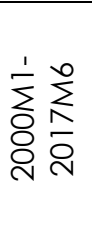 } & EUR & $\begin{array}{c}1.03954 \\
(0.8507) \\
{[0-2]}\end{array}$ & $\begin{array}{c}0.45693 \\
(0.6761) \\
{[0-2]}\end{array}$ & $\begin{array}{c}0.70976 \\
(0.7611) \\
{[0-13]}\end{array}$ & $\begin{array}{c}-0.43050 \\
(0.3334) \\
{[0-13]}\end{array}$ & $\begin{array}{c}0.93165 \\
(0.8242) \\
{[0-2]}\end{array}$ & $\begin{array}{c}0.14660 \\
(0.5583) \\
{[0-2]}\end{array}$ \\
\hline & USD & $\begin{array}{c}2.31080 \\
(0.9896) \\
{[0-2]}\end{array}$ & $\begin{array}{c}1.59000 \\
(0.9441) \\
{[0-2]}\end{array}$ & $\begin{array}{c}1.74017 \\
(0.9591) \\
{[0-12]}\end{array}$ & $\begin{array}{c}1.09056 \\
(0.8623) \\
{[0-12]}\end{array}$ & $\begin{array}{c}1.98203 \\
(0.9763) \\
{[0-12]}\end{array}$ & $\begin{array}{c}1.10620 \\
(0.8657) \\
{[0-12]}\end{array}$ \\
\hline \multirow{2}{*}{ 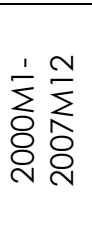 } & EUR & $\begin{array}{c}0.13163 \\
(0.5524) \\
{[0-1]} \\
\end{array}$ & $\begin{array}{c}1.09463 \\
(0.8632) \\
{[0-1]} \\
\end{array}$ & $\begin{array}{c}-1.46082 \\
(0.0720) \\
{[0-11]} \\
\end{array}$ & $\begin{array}{l}1.32892 \\
(0.9081) \\
{[0-11] 11.0}\end{array}$ & $\begin{array}{c}-0.90793 \\
(0.1820) \\
{[0-9]}\end{array}$ & $\begin{array}{c}0.77274 \\
(0.7802) \\
{[0-9]}\end{array}$ \\
\hline & USD & $\begin{array}{c}4.49915 \\
(1.0000) \\
{[0-4]}\end{array}$ & $\begin{array}{c}1.67883 \\
(0.9534) \\
{[0-4]}\end{array}$ & $\begin{array}{c}4.46688 \\
(1.0000) \\
{[0-11]}\end{array}$ & $\begin{array}{c}2.40845 \\
(0.9920) \\
{[0-10]}\end{array}$ & $\begin{array}{c}4.03692 \\
(1.0000) \\
{[0-4]}\end{array}$ & $\begin{array}{c}2.47598 \\
(0.9934) \\
{[0-10]}\end{array}$ \\
\hline \multirow{2}{*}{ 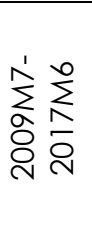 } & EUR & $\begin{array}{c}-1.63529 \\
(0.0510) \\
{[0-1]} \\
\end{array}$ & $\begin{array}{c}-0.82200 \\
(0.2055) \\
{[0-1]} \\
\end{array}$ & $\begin{array}{c}-1.97135 \\
(0.0243) \\
{[0-10]} \\
\end{array}$ & $\begin{array}{c}-2.89484 \\
(0.0019) \\
{[0-10]}\end{array}$ & $\begin{array}{c}-1.77451 \\
(0.0380) \\
{[0-1]} \\
\end{array}$ & $\begin{array}{c}-1.34098 \\
(0.0900) \\
{[0-1]}\end{array}$ \\
\hline & USD & $\begin{array}{c}-0.77192 \\
(0.2201) \\
{[0-3]}\end{array}$ & $\begin{array}{c}0.86019 \\
(0.8052) \\
{[0-2]}\end{array}$ & $\begin{array}{c}-0.94346 \\
(0.1727) \\
{[0-11]}\end{array}$ & $\begin{array}{c}1.66274 \\
(0.9518) \\
{[0-9]}\end{array}$ & $\begin{array}{c}-0.67884 \\
(0.2486) \\
{[0-6]}\end{array}$ & $\begin{array}{c}0.67436 \\
(0.7500) \\
{[0-3]}\end{array}$ \\
\hline
\end{tabular}


Table 3 exhibit no confirmation of PPP in the whole observed period regardless of the reference currency and the method applied. Moreover, in the case of USD as reference currency the results are the same also for both subsamples, not confirming PPP. When the reference currency is EUR, the results are partially supportive for PPP in the post-crisis period, as in three cases the null of a unit root is rejected at $5 \%$ significance level and in two cases at $10 \%$ significance level. While in the pre-crisis period the results show a limited sign of PPP as the null is rejected at $10 \%$ significance level only in one out of six various methodological cases.

Tables 4 and 5 presents $X^{2}$ and $Z$ statistics determined by the ADF test, while Tables 6 and 7 display $X^{2}$ and $Z$ statistics subject to the Phillips-Perron PP individual unit root tests. There is no evidence for PPP when testing the null of a unit root by the Fisher type ADF tests and the Fisher type PP tests for the USD based exchange rates. While for EUR, there is modest support for PPP in the post-crisis period provided by Maddala and $W u X^{2}$ statistic based of the Fisher ADF tests and strong support in the same period given by Choi Z statistic based on the Fisher ADF tests.

Table 4 Results of the Fisher ADF tests (Maddala and Wu X² statistic)

\begin{tabular}{|c|c|c|c|c|c|c|c|}
\hline \multirow{3}{*}{ 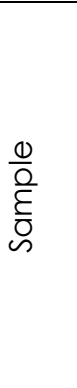 } & \multirow{3}{*}{ 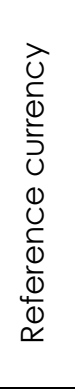 } & \multicolumn{2}{|c|}{$\begin{array}{c}\text { Schwarz information } \\
\text { criterion }\end{array}$} & \multicolumn{2}{|c|}{$\begin{array}{l}\text { Akaike information } \\
\text { criterion }\end{array}$} & \multicolumn{2}{|c|}{$\begin{array}{c}\text { Hannan-Qiunn information } \\
\text { criterion }\end{array}$} \\
\hline & & $\begin{array}{l}\text { Individual } \\
\text { effects }\end{array}$ & \begin{tabular}{|c|} 
Individual \\
effects and \\
individual linea \\
trends
\end{tabular} & $\begin{array}{l}\text { Individual } \\
\text { effects }\end{array}$ & $\begin{array}{l}\text { Individual } \\
\text { effects and } \\
\text { individual } \\
\text { linear trends }\end{array}$ & $\begin{array}{l}\text { Individual } \\
\text { effects }\end{array}$ & $\begin{array}{l}\text { Individual } \\
\text { effects and } \\
\text { individual } \\
\text { linear trends }\end{array}$ \\
\hline & & $\begin{array}{c}\mathrm{X}^{2} \\
\text { (p-value) } \\
\text { [no. of lags] }\end{array}$ & $\begin{array}{c}x^{2} \\
\text { (p-value) } \\
\text { [no. of lags] }\end{array}$ & $\begin{array}{c}x^{2} \\
\text { (p-value) } \\
\text { [no. of } \\
\text { lags] }\end{array}$ & $\begin{array}{c}\mathrm{x}^{2} \\
\text { (p-value) } \\
\text { [no. of lags] }\end{array}$ & $\begin{array}{c}x^{2} \\
\text { (p-value) } \\
\text { [no. of } \\
\text { lags] }\end{array}$ & $\begin{array}{c}\mathrm{x}^{2} \\
\text { (p-value) } \\
\text { [no. of lags] }\end{array}$ \\
\hline \multirow{2}{*}{ 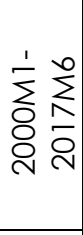 } & EUR & $\begin{array}{c}15.6973 \\
(0.9432) \\
{[0-2]}\end{array}$ & $\begin{array}{c}18.4679 \\
(0.8581) \\
{[0-2]}\end{array}$ & $\begin{array}{c}17.0436 \\
(0.9078) \\
{[0-13]}\end{array}$ & $\begin{array}{c}22.6013 \\
(0.6554) \\
{[0-13]}\end{array}$ & $\begin{array}{c}15.9052 \\
(0.9385) \\
{[0-2]}\end{array}$ & $\begin{array}{c}19.8690 \\
(0.7977) \\
{[0-2]}\end{array}$ \\
\hline & USD & $\begin{array}{c}9.92658 \\
(0.9981) \\
{[0-2]}\end{array}$ & $\begin{array}{c}12.7266 \\
(0.9863) \\
{[0-2]}\end{array}$ & $\begin{array}{c}12.4588 \\
(0.9883) \\
{[0-12]}\end{array}$ & $\begin{array}{c}14.4051 \\
(0.9673) \\
{[0-12]}\end{array}$ & $\begin{array}{c}10.7163 \\
(0.9964) \\
{[0-12]}\end{array}$ & $\begin{array}{c}14.3034 \\
(0.9688) \\
{[0-12]}\end{array}$ \\
\hline \multirow{2}{*}{ 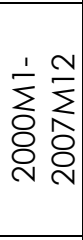 } & EUR & $\begin{array}{c}27.9347 \\
(0.3617) \\
{[0-1]}\end{array}$ & $\begin{array}{c}22.1696 \\
(0.6794) \\
{[0-1]}\end{array}$ & $\begin{array}{c}35.8090 \\
(0.0952) \\
{[0-11]}\end{array}$ & $\begin{array}{c}20.7037 \\
(0.7571) \\
{[0-11]}\end{array}$ & $\begin{array}{c}33.3012 \\
(0.1535) \\
{[0-9]}\end{array}$ & $\begin{array}{c}19.8690 \\
(0.7977) \\
{[0-2]}\end{array}$ \\
\hline & USD & $\begin{array}{c}10.5405 \\
(0.9969) \\
{[0-4]}\end{array}$ & $\begin{array}{c}23.7538 \\
(0.5900) \\
{[0-4]} \\
\end{array}$ & $\begin{array}{c}9.04564 \\
(0.9992) \\
{[0-11]}\end{array}$ & $\begin{array}{c}23.9451 \\
(0.5791) \\
{[0-10]}\end{array}$ & $\begin{array}{c}10.8614 \\
(0.9960) \\
{[0-4]}\end{array}$ & $\begin{array}{c}23.7544 \\
(0.5900) \\
{[0-10]}\end{array}$ \\
\hline \multirow{2}{*}{ 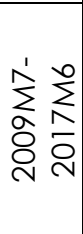 } & EUR & $\begin{array}{c}34.5921 \\
(0.1208) \\
{[0-1]}\end{array}$ & $\begin{array}{c}27.3354 \\
(0.3919) \\
{[0-1]}\end{array}$ & $\begin{array}{c}37.3366 \\
(0.0697) \\
{[0-10]}\end{array}$ & $\begin{array}{c}46.0716 \\
(0.0090) \\
{[0-10]}\end{array}$ & $\begin{array}{c}35.6548 \\
(0.0982) \\
{[0-1]}\end{array}$ & $\begin{array}{c}31.5007 \\
(0.2101) \\
{[0-1]}\end{array}$ \\
\hline & USD & $\begin{array}{c}32.0307 \\
(0.1921) \\
{[0-3]}\end{array}$ & $\begin{array}{c}22.7241 \\
(0.6485) \\
{[0-2]}\end{array}$ & $\begin{array}{c}33.0297 \\
(0.1612) \\
{[0-11]}\end{array}$ & $\begin{array}{c}18.5280 \\
(0.8557) \\
{[0-9]}\end{array}$ & $\begin{array}{c}31.4641 \\
(0.2115) \\
{[0-6]}\end{array}$ & $\begin{array}{c}22.1662 \\
(0.6796) \\
{[0-3]}\end{array}$ \\
\hline
\end{tabular}


Table 5 Results of the Fisher ADF tests (Choi Z statistic)

\begin{tabular}{|c|c|c|c|c|c|c|c|}
\hline \multirow{3}{*}{$\begin{array}{l}\frac{0}{0} \\
\frac{\hat{\varepsilon}}{0} \\
\stackrel{\sim}{n}\end{array}$} & \multirow{3}{*}{ 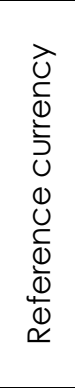 } & \multicolumn{2}{|c|}{$\begin{array}{l}\text { Schwarz information } \\
\text { criterion }\end{array}$} & \multicolumn{2}{|c|}{ Akaike information criterion } & \multicolumn{2}{|c|}{$\begin{array}{l}\text { Hannan-Qiunn information } \\
\text { criterion }\end{array}$} \\
\hline & & $\begin{array}{l}\text { Individual } \\
\text { effects }\end{array}$ & $\begin{array}{c}\text { Individual } \\
\text { effects and } \\
\text { individual linear } \\
\text { trends } \\
\end{array}$ & $\begin{array}{l}\text { Individual } \\
\text { effects }\end{array}$ & $\begin{array}{l}\text { Individual } \\
\text { effects and } \\
\text { individual } \\
\text { linear trends }\end{array}$ & $\begin{array}{l}\text { Individual } \\
\text { effects }\end{array}$ & $\begin{array}{l}\text { Individual } \\
\text { effects and } \\
\text { individual } \\
\text { linear trends }\end{array}$ \\
\hline & & $\begin{array}{c}z \\
\text { (p-value) } \\
\text { [no. of } \\
\text { lags] }\end{array}$ & $\begin{array}{c}\text { Z } \\
\text { (p-value) } \\
\text { [no. of lags] }\end{array}$ & $\begin{array}{c}\mathrm{Z} \\
\text { (p-value) } \\
\text { [no. of } \\
\text { lags] }\end{array}$ & $\begin{array}{c}\text { Z } \\
\text { (p-value) } \\
\text { [no. of lags] }\end{array}$ & $\begin{array}{c}z \\
\text { (p-value) } \\
\text { [no. of } \\
\text { lags] }\end{array}$ & $\begin{array}{c}\text { Z } \\
\text { (p-value) } \\
\text { [no. of lags] }\end{array}$ \\
\hline$\frac{1}{\sum} \sum^{\infty}$ & EUR & $\begin{array}{c}1.14002 \\
(0.8729) \\
{[0-2]}\end{array}$ & $\begin{array}{c}0.51988 \\
(0.6984) \\
{[0-2]}\end{array}$ & $\begin{array}{c}0.89715 \\
(0.8152) \\
{[0-13]}\end{array}$ & $\begin{array}{c}-0.33402 \\
(0.3692) \\
{[0-13]}\end{array}$ & $\begin{array}{c}1.04380 \\
(0.8517) \\
{[0-2]}\end{array}$ & $\begin{array}{c}0.18994 \\
(0.5753) \\
{[0-2]}\end{array}$ \\
\hline 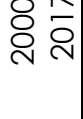 & USD & $\begin{array}{c}2.42817 \\
(0.9924) \\
{[0-2]}\end{array}$ & $\begin{array}{c}1.70512 \\
(0.9559) \\
{[0-2]}\end{array}$ & $\begin{array}{c}1.91899 \\
(0.9725) \\
{[0-12]}\end{array}$ & $\begin{array}{c}1.25715 \\
(0.8957) \\
{[0-12]}\end{array}$ & $\begin{array}{c}2.16121 \\
(0.9847) \\
{[0-12]}\end{array}$ & $\begin{array}{c}1.24837 \\
(0.8941) \\
{[0-12]}\end{array}$ \\
\hline$\frac{1}{\Sigma} \frac{N}{\Sigma}$ & EUR & $\begin{array}{c}0.17496 \\
(0.5694) \\
{[0-1]}\end{array}$ & $\begin{array}{c}1.16506 \\
(0.8780) \\
{[0-1]}\end{array}$ & $\begin{array}{c}-1.33751 \\
(0.0906) \\
{[0-11]}\end{array}$ & $\begin{array}{c}1.66332 \\
(0.9519) \\
{[0-11]}\end{array}$ & $\begin{array}{c}-0.83209 \\
(0.2027) \\
{[0-9]}\end{array}$ & $\begin{array}{c}0.18994 \\
(0.5753) \\
{[0-2]}\end{array}$ \\
\hline ৪্ণ & USD & $\begin{array}{c}4.26183 \\
(1.0000) \\
{[0-4]}\end{array}$ & $\begin{array}{c}1.57148 \\
(0.9420) \\
{[0-4]}\end{array}$ & $\begin{array}{c}4.37007 \\
(1.0000) \\
{[0-11]}\end{array}$ & $\begin{array}{c}2.20450 \\
(0.9863) \\
{[0-10]}\end{array}$ & $\begin{array}{c}3.87405 \\
(0.9999) \\
{[0-4]}\end{array}$ & $\begin{array}{c}2.26236 \\
(0.9882) \\
{[0-10]}\end{array}$ \\
\hline$\dot{N}^{\prime} \sum^{\infty}$ & EUR & $\begin{array}{c}-1.65024 \\
(0.0494) \\
{[0-1]}\end{array}$ & $\begin{array}{c}-0.84615 \\
(0.1987) \\
{[0-1]}\end{array}$ & $\begin{array}{c}-1.95094 \\
(0.0255) \\
{[0-10]}\end{array}$ & $\begin{array}{c}-2.79457 \\
(0.0026) \\
{[0-10]}\end{array}$ & $\begin{array}{c}-1.80148 \\
(0.0358) \\
{[0-1]}\end{array}$ & $\begin{array}{c}-1.38471 \\
(0.0831) \\
{[0-1]}\end{array}$ \\
\hline ঠి & USD & $\begin{array}{c}-0.72903 \\
(0.2330) \\
{[0-3]}\end{array}$ & $\begin{array}{c}0.82276 \\
(0.7947) \\
{[0-2]}\end{array}$ & $\begin{array}{c}-0.80231 \\
(0.21112) \\
{[0-11]}\end{array}$ & $\begin{array}{c}1.75534 \\
(0.9604) \\
{[0-9]}\end{array}$ & $\begin{array}{c}-0.60290 \\
(0.2733) \\
{[0-6]}\end{array}$ & $\begin{array}{c}0.68480 \\
(0.7533) \\
{[0-3]}\end{array}$ \\
\hline
\end{tabular}

Results based on the Fisher PP tests bring no support for PPP at $5 \%$ significance level also in the case for EUR as the reference currency. Allowing for $10 \%$ significance level, there is some evidence for PPP for all kernel approaches when applying individual effects.

Table 6 Results of the Fisher PP tests (Maddala and Wu $X^{2}$ statistic)

\begin{tabular}{|c|c|c|c|c|c|c|c|}
\hline \multirow{3}{*}{$\frac{\frac{0}{O}}{\frac{\mathrm{O}}{\mathrm{D}}}$} & \multirow{3}{*}{ 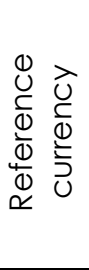 } & \multicolumn{2}{|c|}{ Bartlett kernel } & \multicolumn{2}{|c|}{ Parzen } & \multicolumn{2}{|c|}{ Quadratic spectral kernel } \\
\hline & & $\begin{array}{c}\text { Individual } \\
\text { effects }\end{array}$ & $\begin{array}{l}\text { Individual } \\
\text { effects and } \\
\text { individual } \\
\text { linear trends }\end{array}$ & $\begin{array}{l}\text { Individual } \\
\text { effects }\end{array}$ & $\begin{array}{l}\text { Individual } \\
\text { effects and } \\
\text { individual } \\
\text { linear trends }\end{array}$ & $\begin{array}{l}\text { Individual } \\
\text { effects }\end{array}$ & $\begin{array}{c}\text { Individual } \\
\text { effects and } \\
\text { individual } \\
\text { linear trends }\end{array}$ \\
\hline & & $\begin{array}{c}x^{2} \\
\text { (p-value) }\end{array}$ & $\begin{array}{c}x^{2} \\
\text { (p-value) }\end{array}$ & $\begin{array}{c}x^{2} \\
\text { (p-value) }\end{array}$ & $\begin{array}{c}x^{2} \\
\text { (p-value) }\end{array}$ & $\begin{array}{c}x^{2} \\
\text { (p-value) }\end{array}$ & $\begin{array}{c}x^{2} \\
\text { (p-value) }\end{array}$ \\
\hline \multirow{2}{*}{ 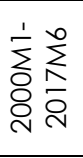 } & EUR & $\begin{array}{l}14.3319 \\
(0.9684) \\
\end{array}$ & $\begin{array}{l}15.1632 \\
(0.9543) \\
\end{array}$ & $\begin{array}{l}14.6903 \\
(0.9627) \\
\end{array}$ & $\begin{array}{l}15.9786 \\
(0.9367) \\
\end{array}$ & $\begin{array}{l}14.5031 \\
(0.9658) \\
\end{array}$ & $\begin{array}{l}16.0037 \\
(0.9361) \\
\end{array}$ \\
\hline & USD & $\begin{array}{l}10.1155 \\
(0.9978) \\
\end{array}$ & $\begin{array}{l}13.8725 \\
(0.9746) \\
\end{array}$ & $\begin{array}{l}10.1149 \\
(0.9978) \\
\end{array}$ & $\begin{array}{l}14.3036 \\
(0.9688) \\
\end{array}$ & $\begin{array}{l}9.85076 \\
(0.9982) \\
\end{array}$ & $\begin{array}{l}13.8674 \\
(0.9747) \\
\end{array}$ \\
\hline \multirow{2}{*}{$\sum_{0}^{1} \frac{N}{\Sigma}$} & EUR & $\begin{array}{l}22.7746 \\
(0.6457) \\
\end{array}$ & $\begin{array}{l}18.1306 \\
(0.8710)\end{array}$ & $\begin{array}{l}22.3688 \\
(0.6684)\end{array}$ & $\begin{array}{r}17.4708 \\
(0.8942)\end{array}$ & $\begin{array}{l}22.5196 \\
(0.6600)\end{array}$ & $\begin{array}{l}17.5680 \\
(0.8909)\end{array}$ \\
\hline & USD & $\begin{array}{l}11.6731 \\
(0.9929)\end{array}$ & $\begin{array}{l}18.4298 \\
(0.8596)\end{array}$ & $\begin{array}{l}11.8016 \\
(0.9922)\end{array}$ & $\begin{array}{l}18.1072 \\
(0.8718)\end{array}$ & $\begin{array}{l}11.8990 \\
(0.9917)\end{array}$ & $\begin{array}{l}18.4599 \\
(0.8584)\end{array}$ \\
\hline \multirow{2}{*}{ 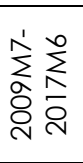 } & EUR & $\begin{array}{l}33.0874 \\
(0.1596) \\
\end{array}$ & $\begin{array}{l}23.9890 \\
(0.5766) \\
\end{array}$ & $\begin{array}{l}33.5547 \\
(0.1466) \\
\end{array}$ & $\begin{array}{l}24.5670 \\
(0.5436) \\
\end{array}$ & $\begin{array}{l}33.4391 \\
(0.1497) \\
\end{array}$ & $\begin{array}{l}24.1601 \\
(0.5668) \\
\end{array}$ \\
\hline & USD & $\begin{array}{l}29.2504 \\
(0.2998) \\
\end{array}$ & $\begin{array}{l}19.0704 \\
(0.8334) \\
\end{array}$ & $\begin{array}{l}29.1760 \\
(0.3031) \\
\end{array}$ & $\begin{array}{l}19.2408 \\
(0.8261) \\
\end{array}$ & $\begin{array}{l}29.0523 \\
(0.3087) \\
\end{array}$ & $\begin{array}{r}19.4913 \\
(0.8150) \\
\end{array}$ \\
\hline
\end{tabular}


Table 7 Results of the Fisher PP tests (Choi Z statistic)

\begin{tabular}{|c|c|c|c|c|c|c|c|}
\hline \multirow{3}{*}{$\frac{\frac{0}{0}}{\frac{\hat{\varepsilon}}{D}}$} & \multirow{3}{*}{ 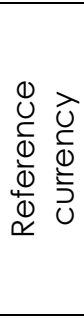 } & \multicolumn{2}{|c|}{ Bartlett kernel } & \multicolumn{2}{|c|}{ Parzen } & \multicolumn{2}{|c|}{ Quadratic spectral kernel } \\
\hline & & $\begin{array}{l}\text { Individual } \\
\text { effects }\end{array}$ & $\begin{array}{l}\text { Individual } \\
\text { effects and } \\
\text { individual } \\
\text { linear } \\
\text { trends }\end{array}$ & $\begin{array}{c}\text { Individual } \\
\text { effects }\end{array}$ & $\begin{array}{l}\text { Individual } \\
\text { effects and } \\
\text { individual } \\
\text { linear trends }\end{array}$ & $\begin{array}{c}\text { Individual } \\
\text { effects }\end{array}$ & $\begin{array}{l}\text { Individual } \\
\text { effects and } \\
\text { individual } \\
\text { linear trends }\end{array}$ \\
\hline & & $\begin{array}{c}\mathrm{Z} \\
\text { (p-value) }\end{array}$ & $\begin{array}{c}\mathrm{Z} \\
\text { (p-value) }\end{array}$ & $\begin{array}{c}\mathrm{Z} \\
\text { (p-value) }\end{array}$ & $\begin{array}{c}\mathrm{Z} \\
\text { (p-value) }\end{array}$ & $\begin{array}{c}\mathrm{Z} \\
\text { (p-value) }\end{array}$ & $\begin{array}{c}\mathrm{Z} \\
\text { (p-value) }\end{array}$ \\
\hline \multirow{2}{*}{ 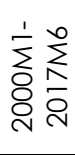 } & EUR & $\begin{array}{l}1.52355 \\
(0.9362) \\
\end{array}$ & $\begin{array}{l}1.20408 \\
(0.8857) \\
\end{array}$ & $\begin{array}{l}1.41205 \\
(0.9210)\end{array}$ & $\begin{array}{l}1.02887 \\
(0.8482) \\
\end{array}$ & $\begin{array}{l}1.46725 \\
(0.9288) \\
\end{array}$ & $\begin{array}{l}1.05864 \\
(0.8551) \\
\end{array}$ \\
\hline & USD & $\begin{array}{l}2.48400 \\
(0.9935)\end{array}$ & $\begin{array}{l}1.52905 \\
(0.9369)\end{array}$ & $\begin{array}{l}2.47947 \\
(0.9964)\end{array}$ & $\begin{array}{l}1.43544 \\
(0.9244)\end{array}$ & $\begin{array}{l}2.54969 \\
(0.9946)\end{array}$ & $\begin{array}{l}1.53299 \\
(0.9347)\end{array}$ \\
\hline \multirow{2}{*}{ 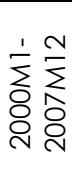 } & EUR & $\begin{array}{l}0.89417 \\
(0.8144)\end{array}$ & $\begin{array}{l}1.71288 \\
(0.9566)\end{array}$ & $\begin{array}{l}0.95467 \\
(0.8301)\end{array}$ & $\begin{array}{l}1.68886 \\
(0.9544)\end{array}$ & $\begin{array}{l}0.94724 \\
(0.8282)\end{array}$ & $\begin{array}{l}1.72866 \\
(0.9581)\end{array}$ \\
\hline & USD & $\begin{array}{l}3.68923 \\
(0.9999)\end{array}$ & $\begin{array}{l}2.13968 \\
(0.9838)\end{array}$ & $\begin{array}{l}3.64279 \\
(0.9999)\end{array}$ & $\begin{array}{l}2.18795 \\
(0.9857)\end{array}$ & $\begin{array}{l}3.72972 \\
(0.9999)\end{array}$ & $\begin{array}{l}2.02797 \\
(0.9787)\end{array}$ \\
\hline \multirow{2}{*}{ 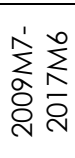 } & EUR & $\begin{array}{r}-1.49616 \\
(0.0673) \\
\end{array}$ & $\begin{array}{r}-0.53076 \\
(0.2978) \\
\end{array}$ & $\begin{array}{r}-1.55914 \\
(0.0595) \\
\end{array}$ & $\begin{array}{r}-0.66179 \\
(0.2541) \\
\end{array}$ & $\begin{array}{r}-1.55069 \\
(0.0605) \\
\end{array}$ & $\begin{array}{r}-0.57210 \\
(0.2836) \\
\end{array}$ \\
\hline & USD & $\begin{array}{r}-0.27977 \\
(0.3898)\end{array}$ & $\begin{array}{l}1.28587 \\
(0.9008)\end{array}$ & $\begin{array}{r}-0.38960 \\
(0.3484)\end{array}$ & $\begin{array}{l}1.19496 \\
(0.8839)\end{array}$ & $\begin{array}{r}-0.35493 \\
(0.3613)\end{array}$ & $\begin{array}{l}1.15615 \\
(0.8762)\end{array}$ \\
\hline
\end{tabular}

Our results are limited to the panel unit root tests of the first generation and are based on the exchange rates of the observed economies in respect to the EUR and the USD. Thus, the prospective work on PPP can be planned in at least two directions. First, the exchange rate theory could be scrutinized with other methodological approaches, such as panel unit root tests that allow for crosssectional dependence, nonlinear behavior, and by testing the mean decline of the real exchange rates and calculating the half-lives of shocks to the real exchange rates in order to estimate the adjustment speed. Second, the robustness of PPP proposition in ASEAN+3 economies could be further tested by taking into account additional benchmark currencies.

\section{Conclusions}

The results from a variety of panel stationarity tests, elaborated in this paper, do not allow us to draw a final verdict on the validity of PPP for Asian economies. The heterogeneity of empirical outcomes notwithstanding, we can emphasize the following main conclusions. First, the PPP is empirically recognized in the whole time span only for EUR-based real exchange rates solely by Levin, Lin and Chu test. Second, in just few cases we were able to detect the mean reversion process of real exchange rates calculated from EUR series in the pre-economic crisis period. Third, the results (based on EUR series) are more in favour of PPP in the post-crisis period. In addition, if we consider the results of the Levin, Lin and Chu test the theory holds for EUR as well as for USD rates. All these cases suggest that for determining the equilibrium exchange rates for the selected Asian countries PPP can serve as a reasonable alternative and simultaneously as an indicator of economic integration of the analysed economies.

\section{References}

1. Arize, A. C., Malindretos, J., Ghosh, D. (2015). Purchasing Power Parity-symmetry and Proportionality: Evidence from 116 Countries. International Review of Economics and Finance, Vol. 37, No. C, pp. 69-85. 
2. Breitung, J. (2000). The Local Power of Some Unit Root Tests for Panel Data. In Advances in Econometrics, Vol. 15: Nonstationary Panels, Panel Cointegration, and Dynamic Panels, Baltagi B. (Ed.), JAl Press, Amsterdam, pp. 161-178.

3. Chang, T., Lee, C. H., Liu, W. C. (2012). Nonlinear Adjustment to Purchasing Power Parity for ASEAN Countries. Japan and the World Economy, Vol. 24, No. 4, pp. 325-331.

4. Chang, T., Zhang, Y., Liu, W. C. (2010). Purchasing Power Parity for ASEAN-8 Countries: Panel SURKSS Tests. Applied Economics Letters, Vol. 17, No. 15, pp. 1517-1523.

5. Choi, I. (2001). Unit Root Tests for Panel Data. Journal for International Money and Finance, Vol. 20, pp. 249-272.

6. Choji, N. M., Sek, S. K. (2017). Testing for Purchasing Power Parity for ASEAN-5 Using Asymmetric Cointegration Tests. International Journal of Advanced and Applied Sciences, Vol. 11, No. 4, pp. 155-159.

7. Fisher, R. A. (1932). Statistical Methods for Research Workers. Oliver\&Boyd, Edinburgh.

8. Froot, K. A., Rogoff, K. (1995). Perspectives on PPP and Long-Run Real Exchange Rates, In Handbook of International Economics Vol. III, Grossman, G., Rogoff, K. (eds.), Elsevier Science, pp. 1647-1688.

9. Hurlin, C. (2010). What Would Nelson and Plosser Find Had They Used Panel Unit Root Tests? Applied Economics, Vol. 42, No. 12, pp. 1515-1531.

10.Im, K. S., Pesaran, M. H., Shin, Y. (2003). Testing for Unit Roots in Heterogenerous Panels. Journal of Econometrics, Vol. 115, No. 1, pp. 53-74.

11.Lau, C. K. M., Suvankulov, F., Su, Y., Chau, F. (2012). Some Cautions on the Use of Nonlinear Panel Unit Root Tests: Evidence from a Modified Series-Specific Non-Linear Panel Unit-Root Test. Economic Modelling, Vol. 29, No. 3, pp. 810-816.

12.Levin, A., Lin, C. F., Chu, C. (2002). Unit Root Testing in Panel Data: Asymptotic and FiniteSample Properties. Journal of Econometrics, Vol. 108, No. 1, pp. 1-24.

13. Lu, Y. R., Chang, T. (2011). Long-Run Purchasing Power Parity with Asymmetric Adjustment: Further Evidence from China. Applied Economics Letters, Vol. 18, No. 9, pp. 881-886.

14.Maddala, G. S., Wu, S. (1999). A Comparative Study of Unit Root Tests with Panel and a New Simple Test. Oxford Bulletin of Economics and Statistics, Vol. 61, pp. 631-653.

15.Munir, Q., Kok, S. C. (2015). Purchasing Power Parity of ASEAN-5 Countries Revisited: Heterogeneity, Structural Breaks and Cross-sectional Dependence. Global Economic Review, Vol. 44, No. 1, pp. 116-149.

16.NBER (2012). US Business Cycle Expansions and Contractions. Available at http://www.nber.org/cycles/US_Business_Cycle_Expansions_and_Contractions_20120423.p df [05 March 2018].

17.Parikh, A., Wakerly, E. (2000). Real Exchange Rates and Unit Root Tests. Weltwirtschaftliches Archiv, Vol. 136, No. 3, pp. 478-490.

18.Zhou, S., Kutan, A. M. (2011). Is the Evidence for PPP Reliable? A Sustainability Examination of the Stationarity of Real Exchange Rates. Journal of Banking \& Finance, Vol. 35, No. 9, pp. 2479-2490.

\section{About the authors}

Darja Boršič, PhD, is a Full Professor in the field of applied economics and econometrics, and an Associate Professor in the field of economic theory and political economy at the Faculty of Economics and Business, University of Maribor. She is the head of the Institute for Economic Diagnosis and Prognosis and an active researcher within the research programme entitled "Adjustment of the Slovenian economy and development identity of Slovenia in the EU«. Her research is focused on price levels, exchange rates and the labour market. In the period from December 2011 to June 2014 and from October 2015 to September 2017, she served as the Vice-dean for international cooperation at the Faculty of Economics and Business, University of Maribor dealing mainly with international accreditation procedures. The author can be contacted at: darja.borsic@um.si. 
Jani Bekö, PhD, is a Full Professor of economic theory and applied economics at the Faculty of Economics and Business, University of Maribor, and the head of the Department of Political Economy. He teaches undergraduate and postgraduate courses on microeconomics, the theory of markets and prices, macroeconomics, international economics and the development of economic theory. He is an active researcher within the research programme entitled $»$ Adjustment of the Slovenian economy and development identity of Slovenia in the EU«. His bibliography is comprised of more than 250 entries. The author can be contacted at: jani.beko@um.si. 